\title{
Highly sensitive detection of methane by near-infrared laser absorption spectroscopy using a compact dense-pattern multipass cell
}

\author{
Kun Liu ${ }^{1}$, Lei Wang ${ }^{1}$, Tu Tan ${ }^{1}$, Guishi Wang ${ }^{1}$, Weijun Zhang ${ }^{1}$, Weidong Chen ${ }^{2}$, Xiaoming Gao ${ }^{1, 冈}$ \\ ${ }^{1}$ Anhui Institute of Optics and Fine Mechanics, Chinese Academy of Sciences, Hefei 230031, China \\ ${ }^{2}$ Laboratoire de Physicochimie de l'Atmosphère, Université du Littoral Côte d'Opale, 189A, Av. \\ Maurice Schumann, 59140 Dunkerque, France \\ \} \text { To whom correspondence should be addressed. E-mail: xmgao@aiofm.ac.cn, liukun@aiofm.ac.cn }
}

\begin{abstract}
Highly sensitive detection of atmospheric methane $\left(\mathrm{CH}_{4}\right)$ was performed by long optical pathlength absorption spectroscopy based on a novel compact dense-pattern multipass cell (DP-MPC) in conjunction with a fiber-coupled distributed feedback diode laser operating at $1.653 \mu \mathrm{m}$. Wavelength modulation spectroscopy approach was used and a minimum detectable concentration $(1 \sigma)$ of $100 \mathrm{ppb}$ was obtained with a lock-in time constant of $1 \mathrm{~ms}$. A measurement precision of $<79 \mathrm{ppb}$ was achieved by average of 5 laser scans in $1 \mathrm{~s}$. This newly developed DP-MPC realized 215 times multiple reflections between two very cheap and robust silver coated concave spherical mirrors separated by a distance of $12 \mathrm{~cm}$ (forming an absorption cell of $280 \mathrm{~cm}^{3}$ volume), offering an effective optical path length of $26.4 \mathrm{~m}$, which is very suitable for applications to trace gas sensing in harsh environment, and weight-limited unmanned aerial vehicle (UAV) - or balloon-embedded field observations.
\end{abstract}

\section{Introduction}

High sensitivity and high precision monitoring of atmospheric methane $\left(\mathrm{CH}_{4}\right)$ concentration has become increasingly important for advanced research on carbon cycle, greenhouse gas budgets and perturbations [1-2]. $\mathrm{CH}_{4}$ is well known as one of the most important greenhouse gases playing an important role in global warming and climate change. Although the concentration of methane in natural air $(\sim 1.8 \mathrm{ppm})$ is roughly 200 times lower than that of $\mathrm{CO}_{2}(\sim 400 \mathrm{ppm})$, methane presents a global warming potential 25 times greater than $\mathrm{CO}_{2}$ and may account for 15 percent of anticipated global warming [3]. Methane is also involved in complex feedback mechanisms in atmospheric chemistry and can serve as makers of large-scale dynamics of the earth's atmosphere [4]. It was recognized that methane emissions originate from both natural sources (such as plants oxidation in anaerobic conditions, oceans, wetlands) and anthropogenic sources (like domestic animals, industries, biomass burning, fossil fuel production, etc) [5]. For instance, irrigated rice cultivation is thought to be a major source of atmospheric $\mathrm{CH}_{4}$ and may constitute 10 to $30 \%$ of the total methane emitted into the atmosphere [6]. The atmospheric methane concentrations have increased from a $700 \mathrm{ppb}$ level to 1.8 ppm since 1750 with a rate of about $1 \%$ per year [3,7], but the growth rate slowed in the $1990 \mathrm{~s}$, the methane burden was nearly constant during the period of 1999-2006. Yet strong growth resumed in 2007. The reasons for these observed changes remain poorly understood because of limited knowledge of its global sources and sinks controlling the global methane budget [1]. Strange craters and burning ice are another examples of mysterious recently happenings on the planet, which are relevant to methane [Scientific American, August 2, 2014]. In addition, methane is a widely used flammable and 
explosive fuel, which can lead to a fatal accident. These important various applications in environmental science, climate change study and industrial monitoring have been a key driving force for the development of robust, sensitive, selective, accurate and precise measurement infrastructure for monitoring $\mathrm{CH}_{4}$ concentration in the atmosphere.

Various techniques have been developed for methane detection. Laser absorption spectroscopy based techniques offer the unique advantages for fast, self-calibration, highly selective and highly sensitive in situ quantification of $\mathrm{CH}_{4}$ without any sample preparation [8-14]. Based on the Beer-Lambert absorption law, the detection sensitivity by absorption spectroscopy is proportional to the molecule absorption path length and the molecule absorption line intensity. In general, sensitive detection scheme is based on using molecular fundamental vibration-rotation transition in the mid-infrared (mid-IR) in conjunction with a traditional multipass cell to achieve the necessary detection sensitivity (of ppb or ppt $[12,13]$ ). Three types of multipass cells are most commonly applied: White cells [15,16], Herriott cells [17], and Chernin [18]. Despite the high detection sensitivity achieved, the sensors based on mid-infrared laser source coupled to a traditional multipass cell present some drawbacks: (1) mid-infrared lasers and detectors are costly compared to the devices operating in the near-IR (despite that their lifetime are not as long as those in the near-IR), (2) optical fiber coupling technology is not yet mature and readily available for realization of cost-effective compact and robust sensor platform. Moreover, for the compact Herriott configuration using conventional design, the number of the light passes inside the cell is limited by the overlapping of light spots on the cell mirrors, which limit the achievable optical path length within a compact cell size. A variation of the Herriott cell, called astigmatic mirror cell has been developed to spread the light spots over the entire mirror surfaces to avoid light beam overlapping [19]. This configuration greatly increases the number of light spots achievable and hence the light pass number in a smaller cell volume than Herriott cell. However, the manufacturing of an astigmatic mirror is more difficult than a concave spherical mirror, which will increase significantly the cost. Recently, sub-ppb detection of $\mathrm{C}_{2} \mathrm{H}_{6}$ using a novel dense-pattern multipass cell (DP-MPC) was reported by Karol Krzempek et al. [20]. The DP-MPC is developed by Sentinel Photonics Inc. [21] offering $57.6 \mathrm{~m}$ effective optical path length with 459 times light passes between two spherical mirrors separated by $13 \mathrm{~cm}, 5$ concentric cycles of light spots were realized on the mirror without light overlapping. Wei Ren et al. also reported high sensitivity detection of $\mathrm{CH}_{4}$ and $\mathrm{N}_{2} \mathrm{O}$ by using the same $57.6 \mathrm{~m}$ ultra-compact multipass gas absorption cell [22]. Such a novel DP-MPC needs however to use dielectric-coated mirror to realize high reflectivity with a wavelength-dependent narrow spectral bandwidth.

In this paper, we report an alternative solution using cheap metal coating to realize a highly compact DP-MPC. The novel DP-MPC consists of two silver coated concave spherical mirrors separated by a distance of $12 \mathrm{~cm}$ and it offers an effective optical pathlength of $26.4 \mathrm{~m}$. Silver (or gold) coated mirror is less sensitive to harsh environmental contamination and requests less attentive maintenance. This low cost, compact and wavelength-independent widely usable DP-MPC is very suitable for various field applications, in particular for gas sensing in harsh environment, and weight-limited unmanned aerial vehicle (UAV) or balloon-embedded observations.

\section{Experimental details}

A schematic diagram of the experimental setup using a compact DP-MPC is shown in Fig. 1. A fiber-coupled distributed feedback (DFB) diode laser (NLK1E5GAAA, NEL) operating at $1.653 \mu \mathrm{m}$ and room temperature was directly connected to the DP-MPC. The laser was tunable from 6040 to 6054 $\mathrm{cm}^{-1}$, suitable for detection of methane by probing one of the strong absorption lines located at 6046.95 $\mathrm{cm}^{-1}$ in the near infrared. The laser current and temperature were controlled with a commercial diode laser controller (ILX Lightwave LDC-3724). Coarse and fine wavelength tunings were performed by 
changing the laser temperature and current, respectively. Wavelength modulation and second harmonic detection was applied to enhance the detection sensitivity [23].

Laser wavelength scan was realized by feeding an external triangular voltage ramp from a function generator (SPF05, NANJING SAMPLE INSTRUMENT TECHNOLOGIES CO., LTD) to the laser diode current which sweeps the laser wavelength back and forth across the absorption line at a rate of 5 Hz. Wavelength modulation was achieved by adding a $11 \mathrm{kHz}$ sine wave to the DFB laser diode current. The sine form wave was supplied by the sinusoidal signal output of a lock-in amplifier (Stanford Research Systems, Model SR 830 DSP). The voltage ramp and the sine wave were combined with a home made adder and then fed to the laser driver. The laser beam was collimated with a fiber-coupled collimator ( $\sim 4.8 \mathrm{~mm}$ ) and subsequently injected into the compact DP-MPC. The output beam was focused on to a photodetector (New Focus, 2011-FC) with a lens ( $\mathrm{f}=40 \mathrm{~mm}$ ). A lock-in amplifier was used for demodulation of the absorption signal from the photodetector at a frequency of $2 f$ (where $f=11$ $k H z$ is the modulation frequency of the sine wave). The time constant of the lock-in amplifier was set to $1 \mathrm{~ms}$ in combination with an $18 \mathrm{~dB}$ /octave slope (leading to a detection bandwidth of $9.375 \mathrm{~Hz}$ ). The demodulated signal was subsequently digitalized by a DAQ card (NI-USB-6212) and displayed on a laptop via a LabVIEW interface. The signal of the photodetector was also acquired for laser power monitoring.

The used compact DP-MPC consisted of two 2" silver coated concave spherical mirrors separated by a distance of $12 \mathrm{~cm}$, which allows laser beam passing 215 times between two spherical mirrors (leading to an effective optical pathlength of $26.4 \mathrm{~m}$ ). The curvature $\mathrm{R}$ of the used spherical mirrors was $100 \mathrm{~mm}$. With a careful optical design and alignment, a novel dense pattern was configured on the mirror with the objective to maximize light reflection number inside the cell while minimizing light spot overlapping to reduce etalon fringe effects. Such silver (or gold) coated spherical mirrors, instead of dielectric-based spherical mirror, offer not only a low cost DP-MPC device, but also a wide spectral response range from the visible to the middle infrared. A home-developed Labview softerware based program was used to determine and optimize optical design of the DP-MPC according to the ray tracing results [24]. Fig. 2 shows a photograph of a red laser beam patterns on one mirror of the DP-MPC, in comparison with the corresponding patterns by the design simulation. A laser power of $11.8 \mathrm{~mW}$ was injected to the DP-MPC, and the output power was measured to be $116 \mu \mathrm{W}$, which allows one to deduce a reflectivity of the mirrors of about $97.8 \%$ at $1.65 \mu \mathrm{m}$. The two mirrors were mounted on an optical board with a dimension of $200 \times 76 \times 10 \mathrm{~mm}^{3}$ and sealed using a quartz glass tube with an inner diameter of $55 \mathrm{~mm}$. The weight of the DP-MPC with body material in stainless was 3.5 $\mathrm{Kg}$. The sample volume of the DP-MPC is $\sim 280 \mathrm{~cm}^{3}$, much smaller than a traditional concave spherical mirror based Herriott or White MPC (of $\sim 700 \mathrm{~cm}^{3}$ with 80 times reflections), it significantly reduces gas sampling time which is a very important issue that should be carefully addressed for sensing of chemically reactive short-lived gas where the gas residence time in absorption cell should be as short as possible.

\section{Measurements and results}

The main objective of the present work was to develop a compact and low cost sensitive $\mathrm{CH}_{4}$ sensor operating at atmospheric pressure. Selection of the absorption line for methane detection was made based on HITRAN04 database [25]. The best $\mathrm{CH}_{4}$ absorption lines within the spectral tuning range of the used $1.653 \mu \mathrm{m}$ diode laser are the $\mathrm{R}_{3}$ triplet of the $2 v_{3}$ band near $\sim 6046.95 \mathrm{~cm}^{-1}$, they have the highest line intensities and are interference free from other molecules (such as $\mathrm{H}_{2} \mathrm{O}$ and $\mathrm{CO}_{2}$ ). These $\mathrm{R}_{3}$ triplet of the $2 v_{3}$ band at $\sim 6046.95 \mathrm{~cm}^{-1}$ were thus selected as the target. According to the HITRAN04 database, the $\mathrm{R}_{3}$ triplet consists of 3 lines: $F_{1}, F_{2}$ and $A_{2}$, which overlap and not resolved even at low pressure under Doppler broadening conditions. The measurement was carried out at ambient pressure, 
(though it may get a better signal to noise ratio when working at a reduced pressure [26], it requests the use of additional mechanical pump and pressure controller, which increases the sensor size and its cost). In the present work, the laser center current $I_{0}$ was set at $57 \mathrm{~mA}$ with a temperature set at $23{ }^{\circ} \mathrm{C}$ for targeting the selected $\mathrm{CH}_{4}$ absorption lines around $6046.95 \mathrm{~cm}^{-1}$. The amplitude of the triangular ramp was $1.5 \mathrm{~V}$ (3 V peak to peak), which scanned the laser diode current from 27 to $87 \mathrm{~mA}(20 \mathrm{~mA} / \mathrm{V})$. Fig. 3 shows a calibration curve of the laser wavelength as a function of the laser current at temperature of $23^{\circ} \mathrm{C}$, and an absorption spectrum of $200 \mathrm{ppm} \mathrm{CH}_{4}$ which was simulated based on HITRAN04 database [25]. The laser wavelength was measured with a wavelength meter (Burleigh, WA-1500).

In wavelength modulation spectroscopy, amplitude of the $2^{\text {nd }}$ harmonic signal is dependent on the modulation amplitude [27]. To determine the optimum modulation amplitude, $2^{\text {nd }}$ harmonic signals of 200 ppm $\mathrm{CH}_{4}$ measured at different modulation amplitude were recorded. The measurement was carried out at normal atmosphere. The $2^{\text {nd }}$ harmonic signal amplitude of $\mathrm{CH}_{4}$ as a function of the modulation amplitude is shown in Fig. 4. The optimum modulation amplitude of the sine wave for $\mathrm{CH}_{4}$ detection was found to be $0.3 \mathrm{~V}$, and the corresponding laser wavelength modulation amplitude was $\Delta v_{\mathrm{m}} \approx 0.15 \mathrm{~cm}^{-1}$. According to the HITRAN04 database, the $200 \mathrm{ppm} \mathrm{CH}_{4}$ line width (HWHM) of the targeted absorption lines was $\Delta v_{\mathrm{D}} \approx 0.068 \mathrm{~cm}^{-1}$. This result agrees with the theoretical optimum modulation amplitude $\Delta v_{\mathrm{m}}=2.2 \Delta v_{\mathrm{D}}$ for wavelength modulation with $2^{\text {nd }}$ harmonic demodulation $[23,27]$. As such a modulation amplitude of $0.3 \mathrm{~V}$ was used in the following measurements. The $2^{\text {nd }}$ harmonic signal is also dependent on the laser power, therefore the signal from the photodetector was recorded for monitoring the laser power fluctuations and the $2 f$ signal amplitude was normalized to the laser power before concentration retrieval.

The response of the $2 f$ signal amplitude to different $\mathrm{CH}_{4}$ concentration levels (diluted in $\mathrm{N}_{2}$ ) was measured and displayed in Fig.5. $\mathrm{CH}_{4}$ concentrations in the range of 7-80 ppm were generated by diluting a 1000 ppm $\mathrm{CH}_{4}: \mathrm{N}_{2}$ calibrated gas mixture sample with pure $\mathrm{N}_{2}$. Two flow meters were used in this calibration process. One flow meter with a flow range of 0-50 ccm was used to control the flow rate of the $1000 \mathrm{ppm} \mathrm{CH}_{4}: \mathrm{N}_{2}$ calibrated gas sample; another one with a flow range of 0-1000 ccm was used to control the flow rate of pure $\mathrm{N}_{2}$. In the measurements, absolute concentration of the diluted $\mathrm{CH}_{4}$ was determined by direct absorption spectroscopy in a $100 \mathrm{~m}$ multipass cell (Aerodyne Research, Inc. model 5612). The $2 f$ signals for each calibrated $\mathrm{CH}_{4}$ concentration were averaged 100 times. The linear response of the $2 f$ signal to $\mathrm{CH}_{4}$ concentration was confirmed by fitting the data with a linear slope as shown in Fig. 5. The R squared value for the linear fit was $>0.99$ with a dynamic measurement range of $\sim 10^{3}$.

For evaluation of the measurement precision and the stability of the compact DP-MPC based $\mathrm{CH}_{4}$ sensor, time series measurements of ambient $\mathrm{CH}_{4}$ sealed in the MPC (so having constant concentration) was performed during 2.5 hours. The measurements were performed by averaging 5 scans with a scan rate of $5 \mathrm{~Hz}$, namely, each point of the signal was obtained in 1 second. 9000 data points were obtained during 2.5 hours' continuous measurement. Histogram plot of measured data is shown in fig. 6, which can be helpful to evaluate the measurement precision. The data distribution is a gaussian profile and the measurement precision, determined by the half width at half maximum (HWHM) was fund to be 79 ppb with $1 \mathrm{~ms}$ lock-in time constant and $1 \mathrm{~s}$ average time. The stability of the compact DP-MPC based $\mathrm{CH}_{4}$ sensor was determined by an Allan analysis. Fig. 7 shows the Allan deviation obtained from the continuous time series measurements. The Allan analysis showed that the sensor allows an averaging time of up to $70 \mathrm{~s}$ and the corresponding minimum detectable concentration of $\mathrm{CH}_{4}$ can be down to 15 ppb. These high sensitivity and high measurement precision illustrate the high performance of the developed compact DP-MPC based $\mathrm{CH}_{4}$ sensor.

Measurement of $\mathrm{CH}_{4}$ in ambient air was performed to evaluate the performance of the developed 
$\mathrm{CH}_{4}$ sensor. Fig. 8 shows the acquired $2 f$ signal of $\mathrm{CH}_{4}$ in the ambient air (without average), the amplitude of the $2 f$ signal is $174 \mu \mathrm{V}$. According to the calibrated curve shown in Fig. 5, the corresponding $\mathrm{CH}_{4}$ concentration is $1.8 \mathrm{ppm}$, in good agreement with the typical ambient value of $\mathrm{CH}_{4}$. The noise level was determined by the standard deviation, deduced from non-absorption wing of the $\mathrm{CH}_{4}$ spectrum, was found to be $\sim 10 \mu \mathrm{V}(1 \sigma)$, which corresponds to a signal-to-noise ratio (SNR) of $\sim 17$. From these results, a minimum detectable concentration of $\mathrm{CH}_{4}$ of $100 \mathrm{ppb}$ was deduced, and a noise equivalent absorption sensitivity (NEAS) of $(1 \sigma) 3.6 \times 10^{-9} \mathrm{~cm}^{-1} \mathrm{~Hz}^{-1 / 2}$ was obtained at atmospheric pressure. Two days' continuous measurements of $\mathrm{CH}_{4}$ in ambient air (Hefei, China) were performed. During the measurement, a dryer and a particle filter were placed before the inlet of the DP-MPC to remove humidity and aerosols. The time series continuous measurements of ambient $\mathrm{CH}_{4}$ concentration are shown in Fig. 9, in which the data were averaged in 1 minute (averaging 300 times laser scans). Measured results shown that the highest $\mathrm{CH}_{4}$ concentration occurs at $\sim 7 \mathrm{am}$, and the lowest concentration occurs at $\sim 11 \mathrm{am}$, respectively. Two consecutive days' measurements represent the same $\mathrm{CH}_{4}$ concentration variation trends. The averaged concentration of a day was found to be $2.01 \mathrm{ppm}$ and 1.99 ppm for a two days' measurement, respectively.

\section{Conclusions}

High sensitivity and precision monitoring of $\mathrm{CH}_{4}$ using a $1.653 \mu \mathrm{m}$ near infrared diode laser was achieved with the help of a novel compact DP-MPC which involved cheap and easily manufactured silver coated concave spherical mirrors to realize a $26.4 \mathrm{~m}$ effective optical pathlength in a cell with a base length of $12 \mathrm{~cm}$. The overall dimension of the $26.4 \mathrm{~m} \mathrm{DP}-\mathrm{MPC}$ is $20 \times 7.6 \times 10.5 \mathrm{~cm}^{3}$ leading to a sample volume of $280 \mathrm{~cm}^{3}$. Furthermore, such metal coated mirror based DP-MPC is less sensitive to harsh environmental contamination and require less attentive maintenance. Such a sensitive, low cost, long lifetime, compact and robust, handheld $\mathrm{CH}_{4}$ sensor is very suitable for application in harsh environment (leaking detection in nature gas industries and coal mines), in agricultural field monitoring and UAV- or balloon-embedded observation.

In our demonstration experience, a minimum detectable concentration of $100 \mathrm{ppb}$ for $\mathrm{CH}_{4}$ detection was obtained with a lock in time constant of $1 \mathrm{~ms}$. A measurement precision of $79 \mathrm{ppb}$ was obtained in 1 second (Averaging 5 times of laser scans). The detection sensitivity can be still improved by further averaging the data, locking the laser wavelength to the targeted absorption line center and increasing time constant of lock-in amplifier, or use filter technique like Kalman Filter [28, 29]. Such DP-MPC based on silver (or gold) coated spherical mirrors is operational in a wide spectral response range which allows one to couple the DP-MPC to quantum cascade laser in the mid-IR.

\section{Acknowledgments}

The authors gratefully acknowledge the financial support from the National Science Foundation of China (No. 41175036, No. 41205120, No. 21307136 and No. 41475023). The support of the French

Agence Nationale de la Recherche (ANR) under the NexCILAS contract (BLANC 2011) is acknowledged.

\section{References}

[1] E. G. Nisbet, E. J. Dlugokencky, P. Bousquet, Methane on the Rise-Again, Science 343 (2014) 493-495 
[2] D. R. Caulton, P. B. Shepson, R. L. Santoro, J. P. Sparks, R. W. Howarth, A. R. Ingraffea, M. O. L. Cambaliza, C. Sweeney, A. Karion, K. J. Davis, B. H. Stirm, S. A. Montzka, and B. R. Miller, Toward a better understanding and quantification of methane emissions from shale gas development, PNAS 111 (2014) 6237-6242

[3] H. Rodhe, A Comparison of the Contribution of Various Gases to the Greenhouse Effect, Science 248 (1990) 1217-1219

[4] I. S. A. Isaksen, M. Gauss, G. Myhre, K. M. Anthony, and C. Ruppel, Strong atmospheric chemistry feedback to climate warming from Arctic methane emissions, Global Biogeochem. Cycles 25 (2011) GB2002

[5] S. M. Miller, S. C. Wofsy, A. M. Michalak, E. A. Kort, A. E. Andrews, S. C. Biraud, E. J. Dlugokencky, J. Eluszkiewicz, M. L. Fischer, G. J. Maenhout, B. R. Miller, J. B. Miller, S. A. Montzka, T. Nehrkorn, and C. Sweeney, Anthropogenic emissions of methane in the United States, PNAS 110 (2013) 20018-20022

[6] J.T. Houghton, G.J. Jenkins, J.J. Ephraums, Climate Change, The IPCC Scientific Assessment 3, Cambridge Univ. Press, New York, (1990)

[7] S. Solomon, D. Qin, M. Manning, Z. Chen, M. Marquis, K. B. Averyt, M. Tignor and H. L. Miller, (Eds.) (2007). Contribution of Working Group I to the Fourth Assessment Report of the Intergovernmental Panelon Climate Change. Cambridge, United Kingdom and New York, NY, USA: Cambridge University Press.

[8] E.R. Crosson, A cavity ring-down analyzer for measuring atmospheric levels of methane, carbon dioxide, and water vapor, Appl. Phys. B 92 (2008) 403-408

[9] E. S.F. Berman, M. Fladeland, J. Liem, R. Kolyer, M. Gupta, Greenhouse gas analyzer for measurements of carbon dioxide, methane, and water vapor aboard an unmanned aerial vehicle, Sensors and Actuators B: Chemical 169 (2012) 128-135

[10] A. Grossel, V. Zeninari, B. Parvitte, L. Joly, D. Courtois, Optimization of a compact photoacoustic quantum cascade laser spectrometer for atmospheric flux measurements: application to the detection of methane and nitrous oxide, Appl. Phys. B 88 (2007) 483-492

[11] M. Jahjah, W. Ren, P. Stefański, R. Lewicki, J. Zhang, W. Jiang, J. Tarka, F.K. Tittel, A compact QCL based methane and nitrous oxide sensor for environmental and medical applications, Analyst 139 (2014) 2065-2069

[12] D.D. Nelson, B. McManus, S. Urbanski, S. Herndon, M.S. Zahniser, High precision measurements of atmospheric nitrous oxide and methane using thermoelectrically cooled mid-infrared quantum cascade lasers and detectors, Spectrochim. Acta. A. Mol. Biomol. Spectrosc. 60 (2004) 3325-3335

[13] I. Mappé, L. Joly, G. Durry, X. Thomas, T. Decarpenterie, J. Cousin, N. Dumelie, E. Roth, A. Chakir, P.G. Grillon, A quantum cascade laser absorption spectrometer devoted to the in situ measurement of atmospheric $\mathrm{N}_{2} \mathrm{O}$ and $\mathrm{CH}_{4}$ emission fluxes, Rev. Sci. Instrum. 84 (2013) 023103

[14] H. Yi, W. Chen, A. Vicet, Z. Cao, X. Gao, T. Nguyen, M. Jahjah, Y. Rouillard, L. Nähle, M. Fischer, T-shape microresonator-based quartz-enhanced photoacoustic spectroscopy for ambient methane monitoring using 3.38- $\mu \mathrm{m}$ antimonide distributed feedback laser diode, Appl. Phys. B 116 (2014) 423-428

[15] R. G. Pilston, J.U. White, A long path gas absorption cell, J. Opt. Soc. Am. 44 (1954) 572-573 
[16] J.U. White, Long optical paths of large aperture, J. Opt. Soc. Am. 32 (1942) 285-288

[17] D. Herriot, H. Kogelnik, R. Kompfner, Off-axis paths in spherical mirror interferometers, Appl. Opt. 3 (1964) 523-526

[18] S. M. Chernin, E. G. Barskaya, Optical multi-pass matrix systems, Appl. Opt. 30 (1991) 51-58

[19] J. B. McManus, P. L. Kebabian, M. S. Zahniser, Astigmatic mirror multi-passabsorption cells for long-path length spectroscopy, Appl. Opt. 34 (1995) 3336-3348

[20] K. Krzempek, M. Jahjah, R. Lewicki, P. Stefan'ski, S. So, D. Thomazy, F.K. Tittel, CW DFB RT diode laser-based sensor for trace-gas detection of ethane using a novel compact multipass gas absorption cell, Appl. Phys. B 112 (2013) 461-465

[21] www.sentinelphotonics.com

[22] W. Ren, W. Jiang, F. K. Tittel, Single-QCL-based absorption sensor for simultaneous trace-gas detection of $\mathrm{CH}_{4}$ and $\mathrm{N}_{2} \mathrm{O}$, Appl. Phys. B 117 (2014) 245-251

[23] P. Kluczynski, J. Gustafsson, A. M. Lindberg, O. Axner, Wavelength modulation absorption spectrometry - an extensive scrutiny of the generation of signals, Spectrochimica Acta Part B 56 (2001) 1277-1354

[24] M. Born and E. Wolf, Principles of Optics, 7th ed, Cambridge University Press, United Kingdom (1999)

[25] L.S. Rothman, D. Jacquemart, A. Barbe, D. Chris Benner, M. Birk, L.R. Brown, M.R. Carleer, C. Chackerian, K. Chance, L.H. Coudert, V. Dana, V.M. Devi, J.-M. Flaud, R.R. Gamache, A. Goldman, J.-M. Hartmann, K.W. Jucks, A.G. Maki, J.-Y. Mandin, S.T. Massie, J. Orphal, A. Perrin, C.P. Rinsland, M.A.H. Smith, J. Tennyson, R.N. Tolchenov, R.A. Toth, J. Vande Auwera, P. Varanasi, G. Wagner, The HITRAN 2004 molecular spectroscopic database, Journal of Quantitative Spectroscopy and Radiative Transfer 96 (2005) 139-204

[26] T. Asakaw , N. Kann, and K. Tonokur, Diode Laser Detection of Greenhouse Gases in the Near-Infrared Region by Wavelength Modulation Spectroscopy: Pressure Dependence of the Detection Sensitivity, Sensors 10 (2010) 4686-4699

[27] S. Schilt, L. Thevenaz, P. Robert, Wavelength modulation spectroscopy: combined frequency and intensity laser modulation, Appl. Opt. 42 (2003) 6728-6738

[28] Haris Riris, Clinton B. Carlisle, and Russell E. Warren, Kalman filtering of tunable diode laser spectrometer absorbance measurements, Appl. Opt. 33 (1994) 5506-5508

[29] D.P. Leleux, R. Claps, W. Chen, F.K. Tittel, T.L. Harman, Applications of Kalman filtering to real-time trace gas concentration measurements, Appl. Phys. B 74 (2002) 85-93 


\section{Figure captions}

Fig. 1 Schematic diagram of the developed $\mathrm{CH}_{4}$ sensor (bottom), and photograph of the compact multipass gas absorption cell (top)

Fig. 2 Photograph of the multipass gas absorption cell beam pattern visualized using a red diode laser beam (left) and the corresponding software simulated pattern (right).

Fig. 3 DFB diode laser wavelength as a function of the injection current at a temperature of $23^{\circ} \mathrm{C}$ associated with a simulated absorption spectrum of $20 \mathrm{ppm} \mathrm{CH}_{4}$.

Fig. 4 Measured $2 f$ signal amplitude of $100 \mathrm{ppm} \mathrm{CH}_{4}$ as a function of the laser modulation amplitude.

Fig. 5 Measured $2 f$ signal as a function of the $\mathrm{CH}_{4}$ concentration

Fig. 6 Histogram plot obtained from time series measurements of $2.2 \mathrm{ppm} \mathrm{CH}_{4}$ sealed in the DP-MPC.

Fig. 7 Allan deviation from time series measurements of $\mathrm{CH}_{4}$ sealed in the DP-MPC.

Fig. $82 f$ absorption spectrum of ambient $\mathrm{CH}_{4}$ at normal atmospheric pressure.

Fig. 9 Ambient $\mathrm{CH}_{4}$ concentration variation during two days continuous measurements. 


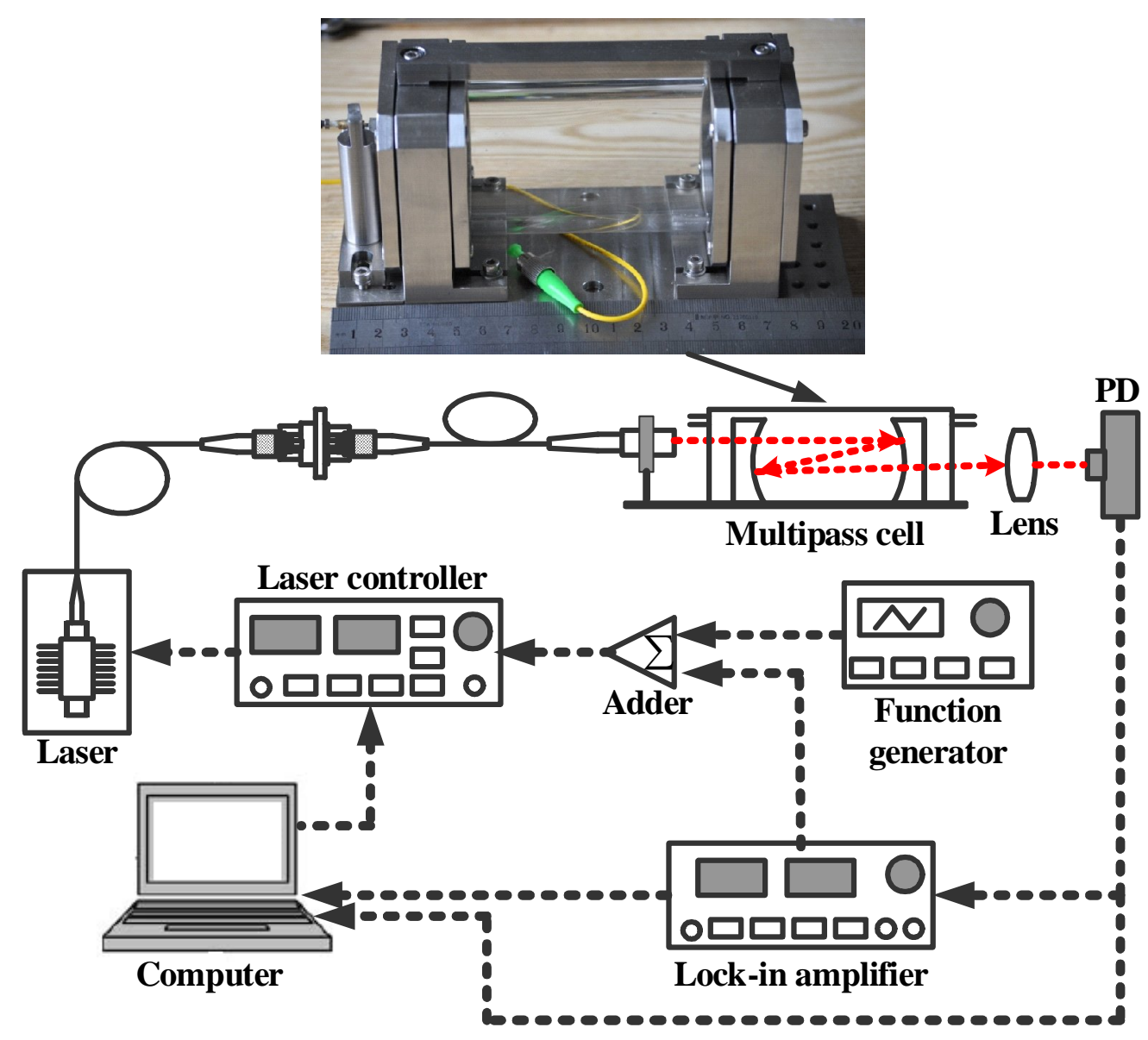

Figure 1 

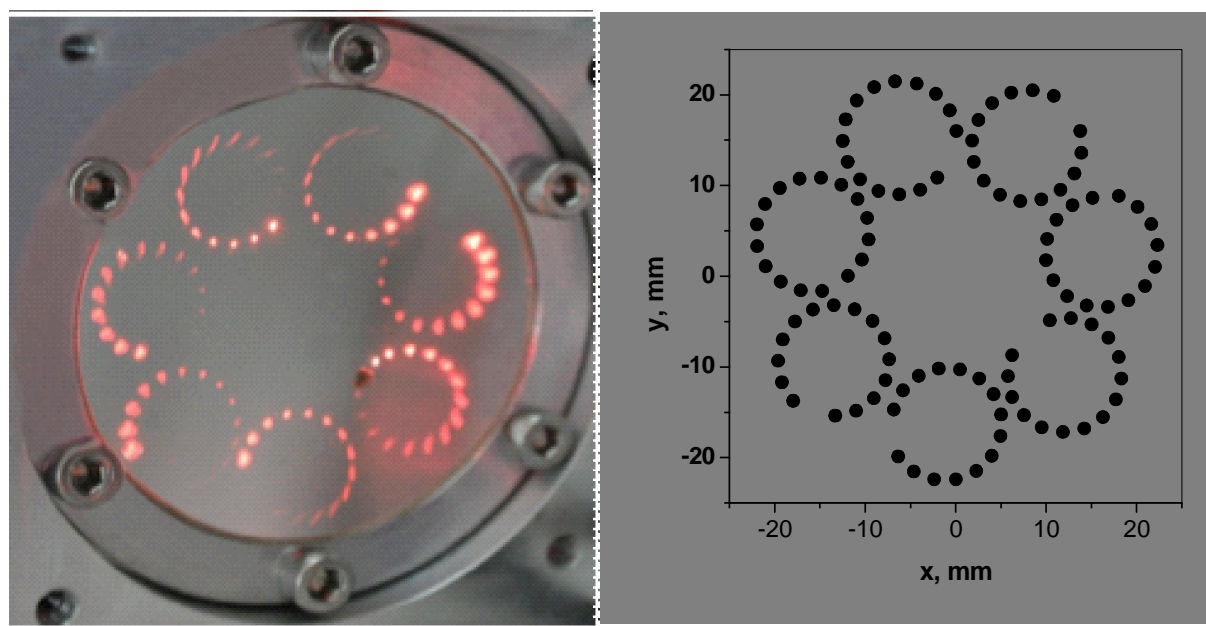

Figure 2 


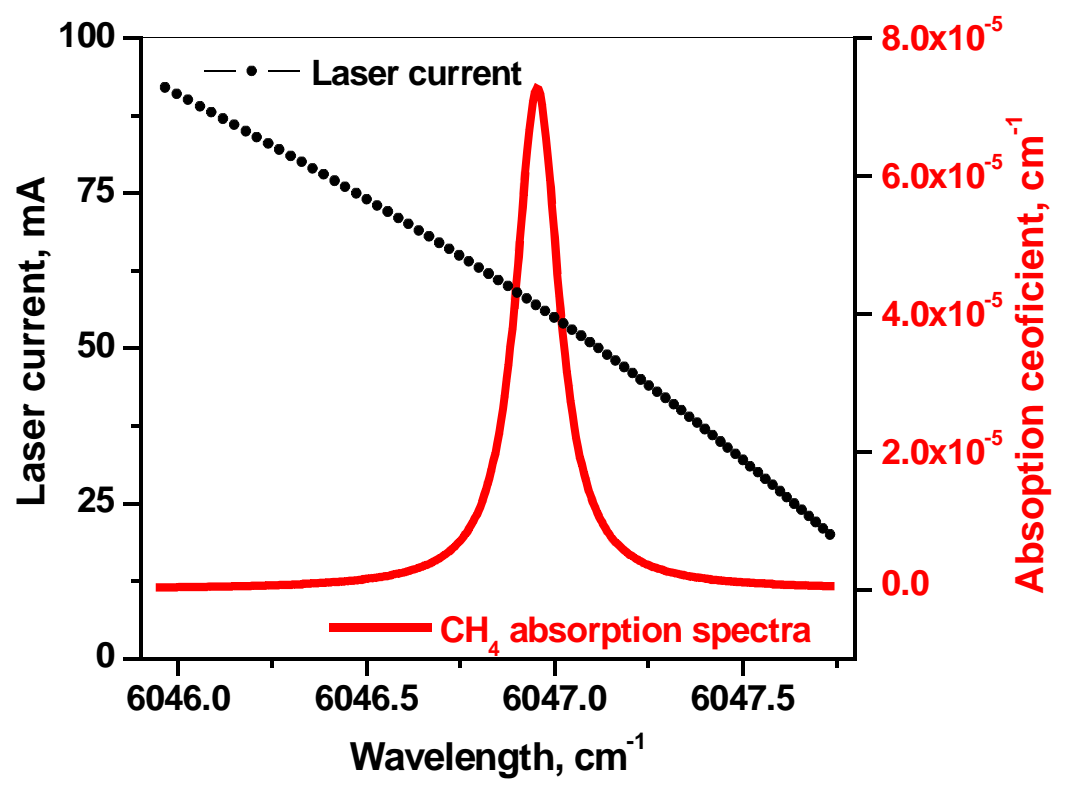

Figure 3 


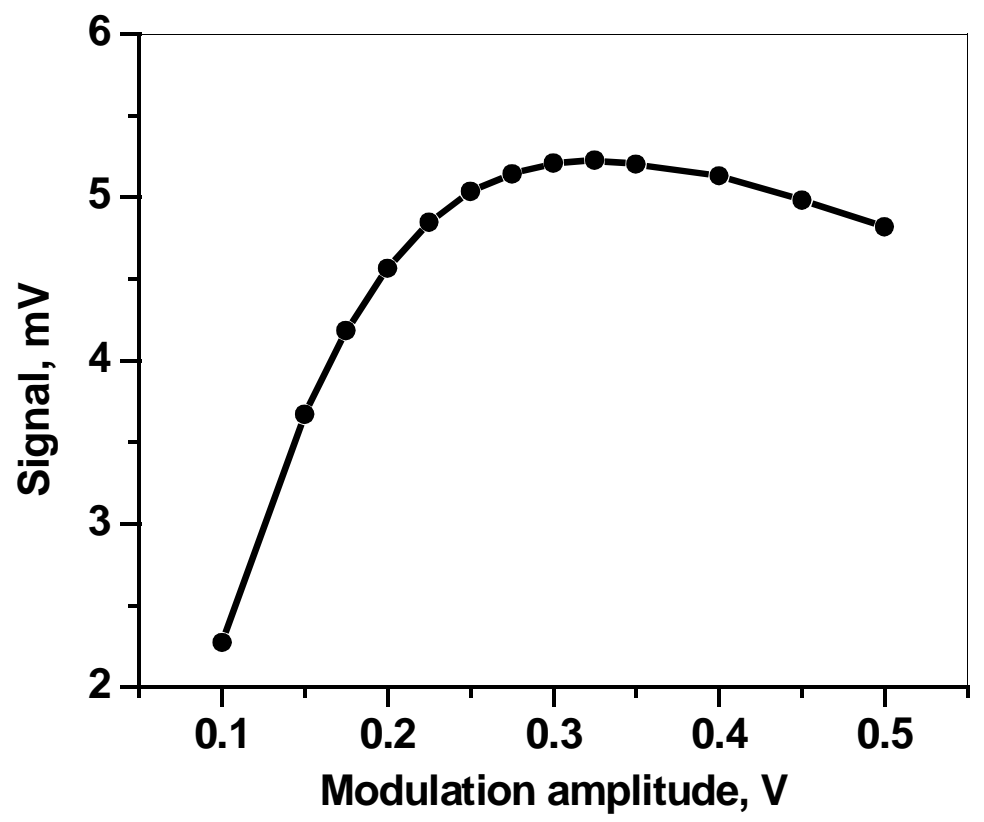

Figure 4 


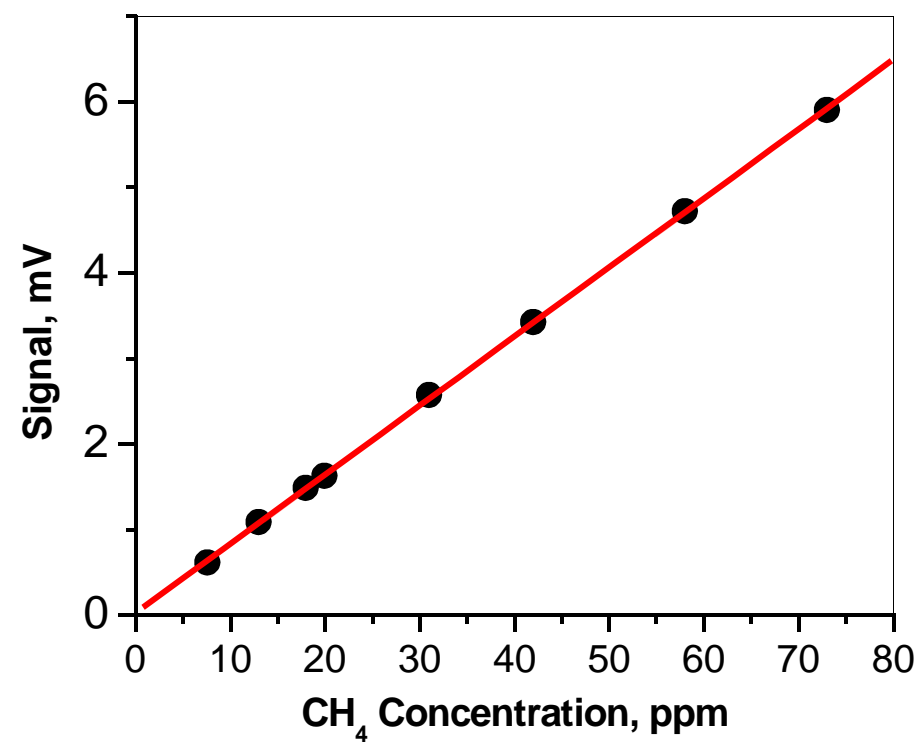

Figure 5 


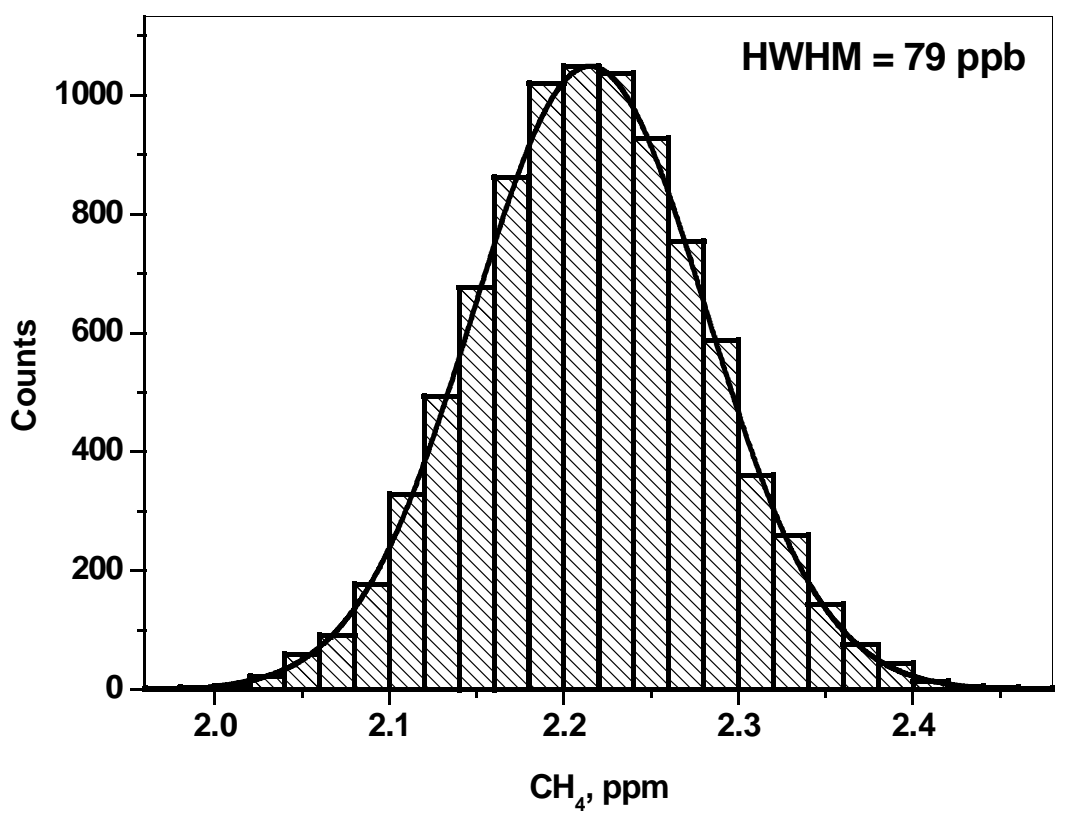

Figure 6 


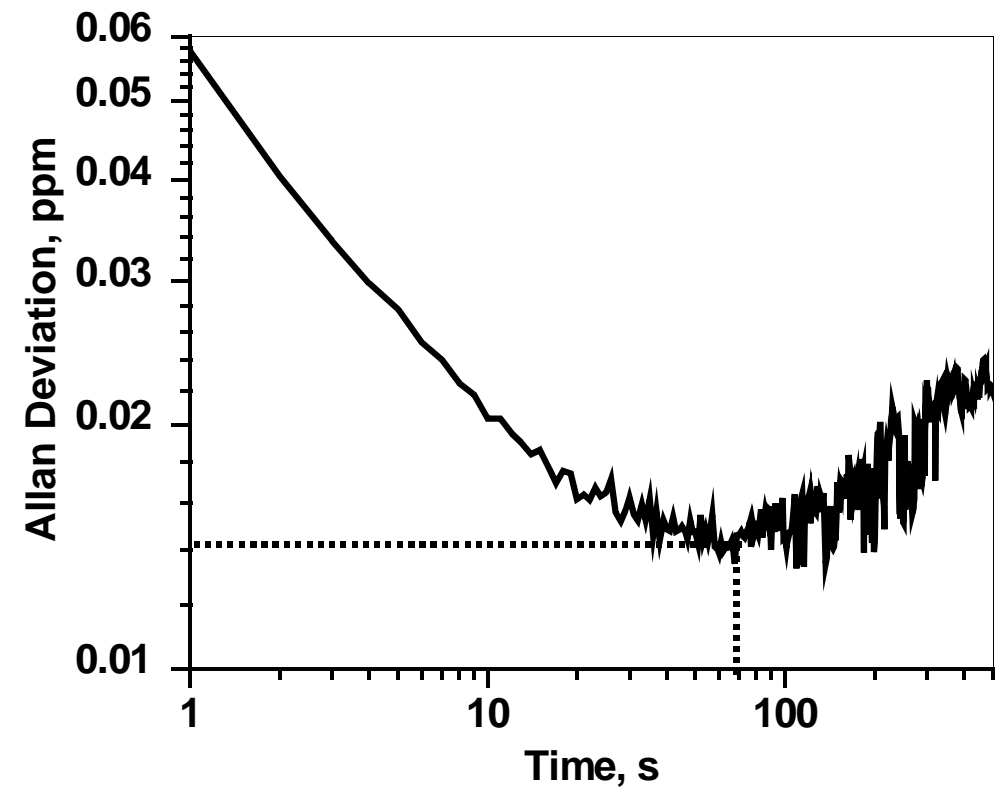

Figure 7 


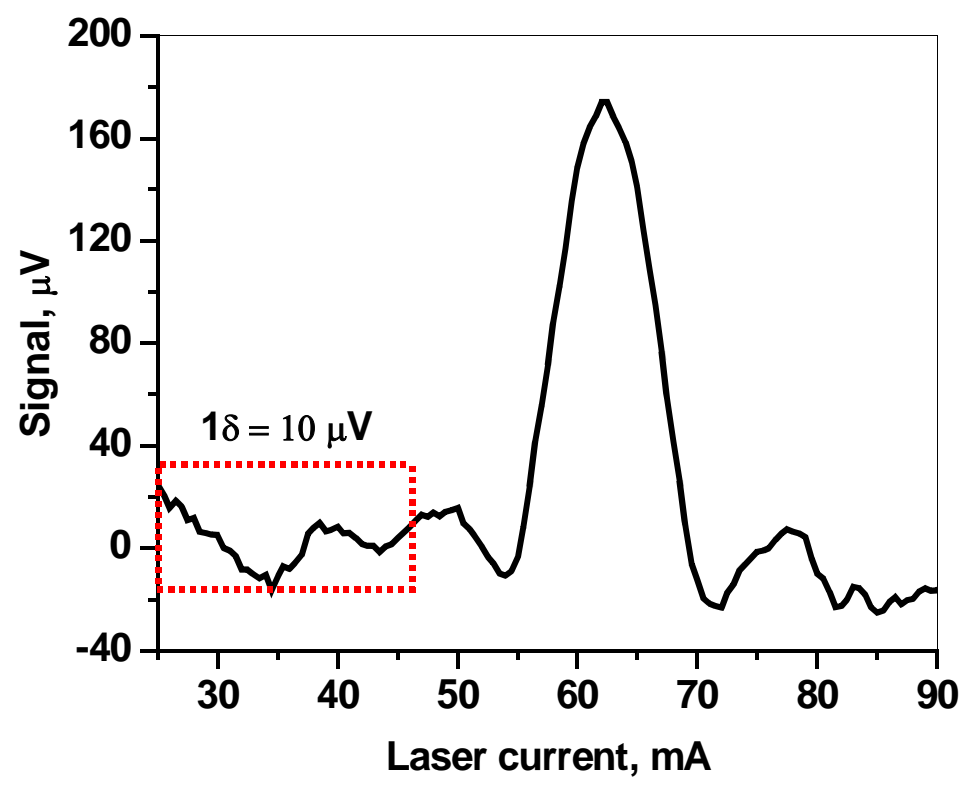

Figure 8 


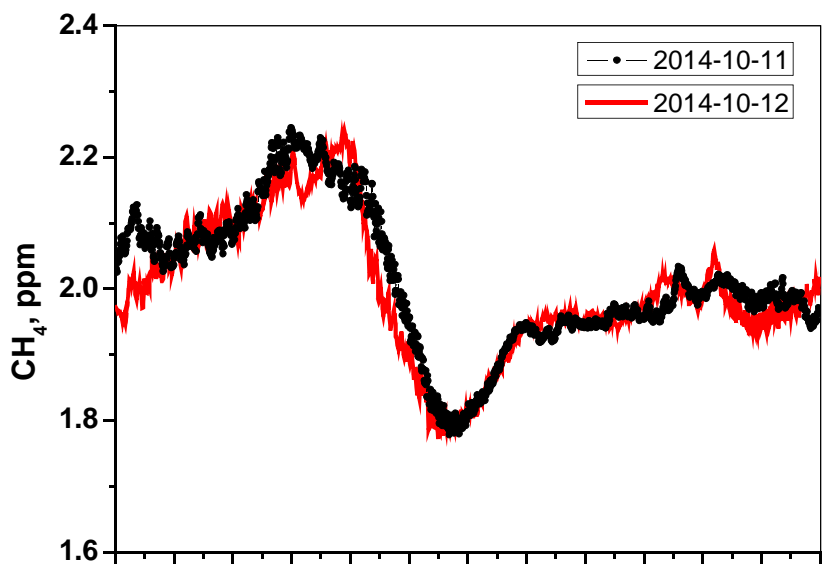

Time of a day, hh:mm

Figure 9 


\section{Author Biographies}

Kun Liu received his Ph.D. degree in optics from Anhui Institute of Optics \& Fine Mechanics, Chinese Academy Sciences in 2010. 2010.10-2011.11, he worked as a post doctor in Rice University, USA. Now he is an associate professor of Anhui Institute of Optics \& Fine Mechanics, Chinese Academy Sciences. His research interests include photoacoustic spectroscopy and laser spectroscopy for application in atmospheric photochemistry and environmental science.

Lei Wang received his Ph.D. degree in optics from Anhui Institute of Optics \& Fine Mechanics, Chinese Academy Sciences in 2010. Now he is an associate professor of Anhui Institute of Optics \& Fine Mechanics, Chinese Academy Sciences. His research interests include optic-electric sensor technology, laser spectroscopy and application.

Tu Tan is a student for Appling Ph.D. degree in optics in Anhui Institute of Optics \& Fine Mechanics, Chinese Academy Sciences. His research interests include laser spectroscopy and laser remote sensor technology for atmospheric science.

Guishi Wang received his Ph.D. degree in optics from Anhui Institute of Optics \& Fine Mechanic s, Chinese Academy Sciences in 2012. Now he is an associate professor of Anhui Institute of Opti cs \& Fine Mechanics, Chinese Academy Sciences. His research interests include laser heterodyne spectroscopy and atmospheric sounding.

Weijun Zhang is Professor at Anhui Institute of Optics \& Fine Mechanics, Chinese Academy of Sciences. He received his Master and Ph.D degree from University of Science and Technology of China and Anhui Institute of Optics \& Fine Mechanics, Chinese Academy Sciences, respectively. The main interests of Prof. Zhang are research on detection methods of trace pollutants, mechanism of environmental pollution, developing laser spectroscopic and mass spectroscopic detection methods for trace organic pollutant, aerosol and important free radical in real-time, and studying the reaction mechanism of free radical in the formation processes of second pollutant.

Weidong Chen received his B.S. in Radio-Physics from Zhangshan University (Guangzhou, China) in 1982, his M.S. and PhD from the University of Sciences \& Technologies of Lille (France) in 1988 and 1991, respectively. From 1991 to 1993 he was an assistant Professor with the University of Sciences \& Technologies of Lille. Since 1993, he has been on the faculty at University of the Littoral Opal Coast as Lecturer, obtained his State PhD (HDR) in 2001 and became full Professor in 2003. His research interests include developments of photonic instrumentation for applied spectroscopy, Optical metrology (concentration, isotope ratio) of trace gases for applications in atmospheric photochemistry and environmental science, Optical parametric laser source generation by frequency conversion and its applications to applied spectroscopy.

Xiaoming Gao is Professor at Anhui Institute of Optics \& Fine Mechanics, Chinese Academy of Sciences. He received his Master and Ph.D degree from Anhui Institute of Optics \& Fine Mechanics, Chinese Academy Sciences in 1993 and 1998, respectively. He studied and worked in Max-Planck- Institute for Quantumoptics, German as postdoctoral from 1998 to 2000. The 
scientific interests of Prof. Gao lie in atmospheric molecules absorption spectroscopy and high sensitive diode laser spectroscopy and application. 